\title{
Effects of intrahepatic cholestasis of pregnancy on hepatic function, changes of inflammatory cytokines and fetal outcomes
}

\author{
LIPING WANG $^{1 *}$, ZHANKAI LU ${ }^{1 *}$, XUEWU ZHOU ${ }^{1}$, YONG DING $^{2}$ and LIJIE GUAN ${ }^{1}$ \\ Departments of ${ }^{1}$ Obstetrics and Gynecology, and ${ }^{2}$ Paediatrics, Daqing Longnan Hospital, \\ Daqing, Heilongjiang 163453, P.R. China
}

Received June 26, 2018; Accepted January 21, 2019

DOI: $10.3892 /$ etm.2019.7312

\begin{abstract}
The effects of intrahepatic cholestasis of pregnancy (ICP) on hepatic function, changes of inflammatory cytokines and fetal outcomes were studied. In total, 663 pregnant women admitted to Daqing Longnan Hospital from July 2016 to December 2017 were selected. There were, 40 cases with ICP enrolled in the observation group, and 40 normal pregnant women were recruited in the normal group. They were also grouped according to hepatic function and inflammatory cytokines, with 40 cases in each group. Neonatal Apgar scores were recorded. The correlations of serum cholylglycine (CG) in pregnant women with umbilical artery systolic-to-diastolic (S/D) ratio in the third trimester of pregnancy, the alanine aminotransferase level, the high-sensitivity C-reactive protein (hs-CRP) level, neonatal Apgar score and gestational week were analyzed. The birth weight in the observation group was lighter than that in the normal group $(\mathrm{P}<0.05)$; the gestational week at birth was earlier than that in the normal group $(\mathrm{P}<0.05)$; Apgar score at birth was lower than that in the normal group $(\mathrm{P}<0.05)$, and the levels of inflammatory cytokines were higher than those in the control group $(\mathrm{P}<0.05)$. Apgar scores of newborns at birth and at 1 and 5 min after birth in the normal hepatic function and normal inflammatory cytokine groups were higher than those in the abnormal hepatic function group $(\mathrm{P}<0.05)$. The serum $\mathrm{CG}$ level in pregnant women was positively correlated with umbilical artery S/D ratio, the alanine aminotransferase level and the hs-CRP level in the third trimester of pregnancy, but negatively correlated with neonatal Apgar score and gestational week. Among patients with ICP, the higher the GG level in the body is, the higher the alanine
\end{abstract}

Correspondence to: Dr Liping Wang, Department of Obstetrics and Gynecology, Daqing Longnan Hospital, 35 Aiguo Road, Daqing, Heilongjiang 163453, P.R. China

E-mail: wangliping515716@126.com

*Contributed equally

Key words: intrahepatic cholestasis of pregnancy, alanine aminotransferase, high-sensitivity C-reactive protein, intrauterine distress, neonatal Apgar score, gestational week aminotransferase, inflammatory cytokine and umbilical artery $\mathrm{S} / \mathrm{D}$ ratio will be, which may cause lower neonatal Apgar score, neonatal asphyxia and premature delivery.

\section{Introduction}

Intrahepatic cholestasis of pregnancy (ICP) is a specific disease during pregnancy, its clinical manifestations include cutaneous pruritus, formation of jaundice and abnormal hepatic function (1). The causes of the disease are not quite clear yet, but this syndrome may severely threaten fetal growth and development, result in intrauterine fetal distress and even intrauterine death, and cause premature delivery, neonatal hypoxia-ischemia and asphyxia (2). Most scholars consider that the pathogenesis of this disease is notably correlated with maternal bile acid level, especially the CG level in the body of pregnant women (3). Long-term high bile acid level, especially high CG level will cause significant spasm in vessels on the surface of placental villi (4), and the increased resistance caused by it will lead to reduced blood flow passing through the area of placenta intervillous space and decreased oxygenation (4), and further bring about insufficient fetal perfusion (5), functional hypoxia-ischemia, difficulty in transshipment of oxygen molecules and nutrient substance from maternal circulation. This will finally result in intrauterine fetal distress and affect growth and development of fetuses (6).

Currently, the treatment for ICP is mainly symptomatic support measures. Fetal development condition is closely monitored, and manual intervention is implemented to terminate pregnancy (7). Its primary mechanism is that long-term high bile acid level, especially high CG level, affects the growth and development of fetuses and may even result in intrauterine fetal distress (8). Although there are many studies on ICP, there are few systematic correlation studies on effects of ICP on hepatic function, changes in the inflammatory cytokine level in the body of pregnant women and fetal outcomes. In order to better answer the national fundamental policy of better prenatal and postnatal care, enhance pregnancy outcomes and improve safety of pregnant and lying-in women and newborns during the perinatal period, this investigation mainly evaluated the deficiencies mentioned above, so as to better guide clinical treatment and improve neonatal outcomes. 


\section{Patients and methods}

General data. A total of 663 pregnant women admitted to Daqing Longnan Hospital (Daqing, China) from July 2016 to December 2017 were selected, and patients complicated with inflammatory diseases of the liver resulted from various factors, gestational diabetes, gestational hypertension, mental disease or abnormal coagulation function, those with thyroid dysfunction during pregnancy, those previously receiving liver- or gall-related operations, or long-term drinkers were excluded. At the same time, according to the diagnostic criteria for ICP in the Obstetrics and Gynecology (7th edition) published in the People's Medical Publishing House (China) (9), there were 40 patients diagnosed with ICP, all of whom suffered from no liver-, gall- or skin-related diseases before pregnancy. After the second and third trimesters of pregnancy, ICP was manifested as significant and persistent pruritus, which often occurred in the proximal hands, feet and limbs, and even mild jaundice was seen. Increased serum cholic acids were found in the biochemical examination. In particular, the index of serum cholylglycine (CG) was more than $30 \mathrm{mg} / \mathrm{l}$, and a mild to moderate increase could be found in bilirubin. The above symptoms and signs returned to normal after pregnancy.

All the patients and their family members signed the inclusion consent, and this study was approved by the Ethics Committee of the Daqing Longnan Hospital. These patients were aged $19-40$ years with an average of $29.5 \pm 0.3$ years. Times of pregnancy: 25 cases of primiparity, 15 cases of re-pregnancy and 10 cases who had previously undergone cesarean section. Additionally, 40 patients with normal pregnancy aged $19-40$ years with a mean age of $29.6 \pm 0.3$ years were selected as the normal group. Times of pregnancy: 26 cases of primiparity, 14 cases of re-pregnancy and 9 cases who had previously undergone cesarean section. There were no statistically significant differences in age, times of pregnancy and whether they had received previous cesarean section between the two groups $(\mathrm{P}>0.05)$. Comparisons of birth weight, gestational week at birth, Apgar score at birth, grade II or above amniotic fluid contamination, intrauterine fetal distress and neonatal jaundice between the two groups are detailed in Table I. All the included patients were divided into two groups according to whether their hepatic function was normal or not. Among them, 40 patients with normal hepatic function aged 19-40 years with a mean age of $29.4 \pm 0.3$ years were included into the normal hepatic function group. Times of pregnancy: 26 cases of primiparity, 14 cases of re-pregnancy and 11 cases who had previously received cesarean section. Forty patients with abnormal hepatic function aged 19-40 years with an average age of $29.4 \pm 0.4$ years were included into the abnormal hepatic function group. Times of pregnancy: 27 cases of primiparity, 13 cases of re-pregnancy and 12 cases who previously had received cesarean section.

Differences in age, times of pregnancy and a history of implementation of cesarean section between the two groups were not statistically significant $(\mathrm{P}>0.05)$. In addition, according to whether inflammatory cytokines [with high-sensitivity C-reactive protein (hs-CRP) as the standard] were increased, the patients were divided into two groups. There were 40 patients with normal inflammatory cytokines aged 19-40 years with an average of $29.5 \pm 0.5$ years included in the normal inflammatory cytokine group. Times of pregnancy: 28 cases of primiparity,
Table I. Comparisons of relevant data between the two groups of newborns (mean $\pm \mathrm{SD}$ ).

\begin{tabular}{lccc}
\hline Variables & $\begin{array}{c}\text { Birth } \\
\text { weight }(\mathrm{g})\end{array}$ & $\begin{array}{c}\text { Gestational } \\
\text { week at } \\
\text { birth (week) }\end{array}$ & $\begin{array}{c}\text { Apgar score } \\
\text { at birth } \\
\text { (point) }\end{array}$ \\
\hline Observation group & $2,156.5 \pm 26.1$ & $34.1 \pm 1.1$ & $6.2 \pm 0.3$ \\
Normal group & $3,158.9 \pm 33.6$ & $38.9 \pm 1.2$ & $8.2 \pm 0.4$ \\
t value & 149.009 & 18.649 & 25.298 \\
P-value & $<0.001$ & $<0.001$ & $<0.001$ \\
\hline
\end{tabular}

12 cases of re-pregnancy and 12 cases who had previously received cesarean section. Forty patients with abnormal inflammatory cytokines aged 19-40 years with an average age of $29.6 \pm 0.5$ years were included into the abnormal inflammatory cytokine group. Times of pregnancy: 29 cases of primiparity, 11 cases of re-pregnancy and 13 cases who had previously received cesarean section. There were no statistically significant differences in age, times of pregnancy and implementation rate of cesarean section between the two groups $(\mathrm{P}>0.05)$.

Intervention methods. All the included patients were given low-flow oxygen, local antipruritic or corticosteroid treatment. Reduction of the concentration of bile acids (ursodeoxycholic acid), vitamin K supplement, vascular dilation, liver protection and other symptomatic support treatments. Regular fetal heart rate monitoring and amniotic fluid index test were carried out. In addition, the levels of bile acid and serum CG in pregnant women and hepatic function of patients were regularly reviewed. Fetal lung maturation was closely monitored, and if necessary, cesarean section was implemented to terminate pregnancy based on the patient's CG level combined with fetal monitoring results. The normal control group was treated with routine pregnancy and delivery.

Observational indexes. The relevant data of newborns such as birth weight, gestational week at birth and Apgar score at birth, intrauterine and postpartum conditions such as the occurrence of grade II or above amniotic fluid contamination, intrauterine fetal distress and the proportion of neonatal jaundice, the levels of inflammation-related cytokines such as hs-CRP, interleukin-6 (IL-6) and tumor necrosis factoralpha (TNF- $\alpha$ ), and Apgar scores at birth, and at 1 and 5 min after birth of newborns delivered by patients with different hepatic functions and different inflammatory cytokine levels were compared between the observation and control groups. The correlation of the level of CG in pregnant women with umbilical artery systolic-to-diastolic (S/D) ratio in the third trimester of pregnancy during antenatal inspection, the alanine aminotransferase level, the hs-CRP level, neonatal Apgar score and gestational week were analyzed.

Evaluation criteria. Apgar score: Apgar score of newborns at birth was assessed in this study. The evaluation criteria included five items: skin color, heart rate pulse, respiratory rate, muscular tone and motor ability, and nerve reflex. The overall score ranged from 0 to 10 points. The total score below 
Table II. Comparison of intrauterine and postpartum conditions between the groups (n).

Grade II or above

Variables

amniotic fluid contamination

Intrauterine fetal distress

Neonatal jaundice

\begin{tabular}{lccc}
\hline Observation group & 29 & 30 & 31 \\
Normal group & 1 & 2 & 1 \\
t value & 38.880 & 37.969 & 43.802 \\
P-value & $<0.001$ & $<0.001$ & $<0.001$ \\
\hline
\end{tabular}

Table III. Comparison of the levels of inflammation-related cytokines between the groups (mean $\pm \mathrm{SD}$ ).

\begin{tabular}{lccc}
\hline Variables & hs-CRP (mg/l) & IL-6 (ng/l) & TNF- $\alpha(\mathrm{ng} / \mathrm{l})$ \\
\hline Observation group & $13.6 \pm 0.7$ & $0.68 \pm 0.1$ & $146.5 \pm 8.3$ \\
Normal group & $8.5 \pm 0.4$ & $0.43 \pm 0.1$ & $83.2 \pm 6.6$ \\
t value & 40.008 & 11.180 & 37.753 \\
P-value & $<0.001$ & $<0.001$ & $<0.001$ \\
\hline
\end{tabular}

7 points represented neonatal asphyxia and that below 4 points severe neonatal asphyxia. Amniotic fluid contamination was divided into 3 grades: grade I with light green amniotic fluid, grade II with dark green or yellow-green amniotic fluid and grade III with brownish yellow and viscous amniotic fluid. Intrauterine fetal distress was diagnosed according to the potential of hydrogen $(\mathrm{pH})$ value of preserved umbilical venous blood during fetal labor to determine the delivery of infants. The $\mathrm{pH}$ value of umbilical venous blood below 7.25 indicated the presence of intrauterine fetal distress. Blood CG $(3.2 \mu \mathrm{g} / \mathrm{ml})$ in pregnant women was detected via the latex-enhanced immunoturbidimetry. The normal value of umbilical artery S/D ratio in the third trimester of pregnancy, i.e., the ratio of endsystolic peak (S) to end-diastolic peak (D) of umbilical artery is generally below 3 . Alanine aminotransferase (0-40 U/l) was detected via Lai colorimetry. Inflammation-related cytokines including hs-CRP (latex-enhanced immunoturbidimetry, normal value in serum $\leq 10 \mathrm{mg} / \mathrm{l}$, cat. no. M020801; So-Fe Biomedicine, Co., Ltd., Shanghai, China), IL-6 (enzyme-linked immunosorbent assay, normal value in serum: 0.37-0.46 ng/l; cat. no. KE00007; ProteinTech Group, Inc., Wuhan, China) and TNF- $\alpha$ (spectrophotometry, normal value in serum: $5-100 \mathrm{ng} / \mathrm{l}$; cat. no. 17590-1-AP; ProteinTech Group, Inc.) were tested.

Statistical analysis. Statistical Product and Service Solutions (SPSS) 21.0 (IBM Corp., Armonk, NY, USA) was adopted for statistical analysis. Measurement data were expressed as mean \pm standard deviation (mean $\pm \mathrm{SD}$ ). The mean values, such as birth weight, gestational week and Apgar score at birth, were compared using the t-test. The percentages, such as grade II or above, amniotic fluid contamination, intrauterine fetal distress and neonatal jaundice percentages were compared between groups using the $\chi^{2}$ test. Spearman's correlation analyses of the serum CG level with umbilical artery S/D ratio in the third trimester of pregnancy during antenatal inspection, the level of alanine aminotransferase, the level of hs-CRP, neonatal Apgar

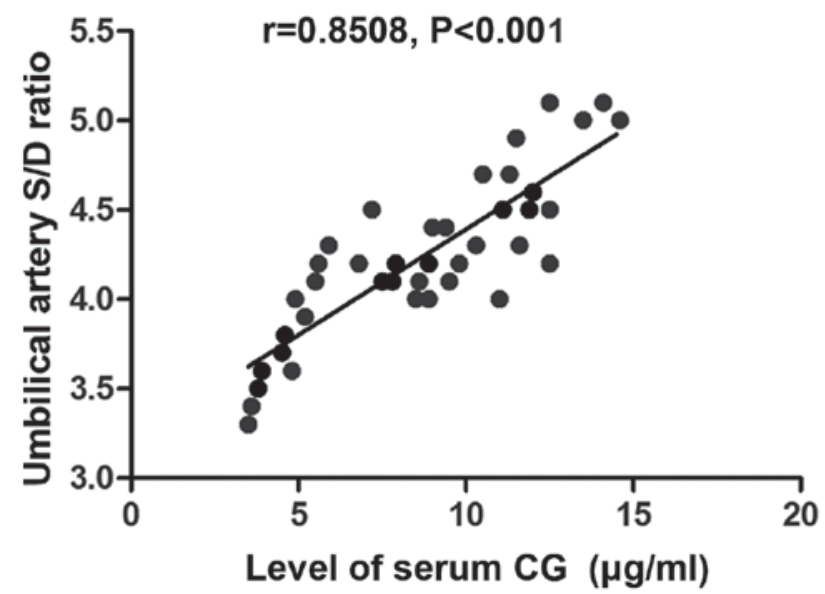

Figure 1. Correlation analysis of the serum CG level in pregnant women with umbilical artery S/D ratio in the third trimester of pregnancy during antenatal inspection. The level of serum CG in pregnant women is positively correlated with umbilical artery $\mathrm{S} / \mathrm{D}$ ratio in the third trimester of pregnancy during antenatal inspection $(\mathrm{P}<0.05)$.

score and gestational week were performed using the correlation coefficient method. $\mathrm{P}<0.05$ was set as the statistically significant difference.

\section{Results}

Comparison of relevant data between the groups of newborns. The birth weight of the observation group was lighter than that of the normal group $(\mathrm{P}<0.05)$, the gestational week at birth was earlier than that of the normal group $(\mathrm{P}<0.05)$, and Apgar score at birth was lower than that of the normal group $(\mathrm{P}<0.05)$ (Table I).

Comparison of intrauterine and postpartum conditions between the groups. The proportions of grade II or above amniotic fluid contamination, intrauterine fetal distress and neonatal jaundice in the observation group were significantly higher than those in the normal group $(\mathrm{P}<0.05)$ (Table II).

Comparison of the levels of inflammation-related cytokines between the groups. The levels of hs-CRP, IL-6 and TNF- $\alpha$ among the inflammation-related cytokines in the observation group detected at the time of inclusion were notably higher than those in the control group $(\mathrm{P}<0.05)$ (Table III).

Changes in Apgar scores of newborns with different hepatic functions. Apgar scores of newborns at birth and at 1 and 
Table IV. Changes in Apgar scores of newborns with different hepatic functions (point, mean \pm SD).

\begin{tabular}{lccc}
\hline Variables & At birth & At 1 min after birth & At 5 min after birth \\
\hline Normal hepatic function group & $8.2 \pm 0.4$ & $8.5 \pm 0.5$ & $9.1 \pm 0.3$ \\
Abnormal hepatic function group & $6.2 \pm 0.3$ & $6.6 \pm 0.4$ & $7.1 \pm 0.2$ \\
t value & 40.008 & 18.767 & 35.082 \\
P-value & $<0.001$ & $<0.001$ & $<0.001$ \\
\hline
\end{tabular}

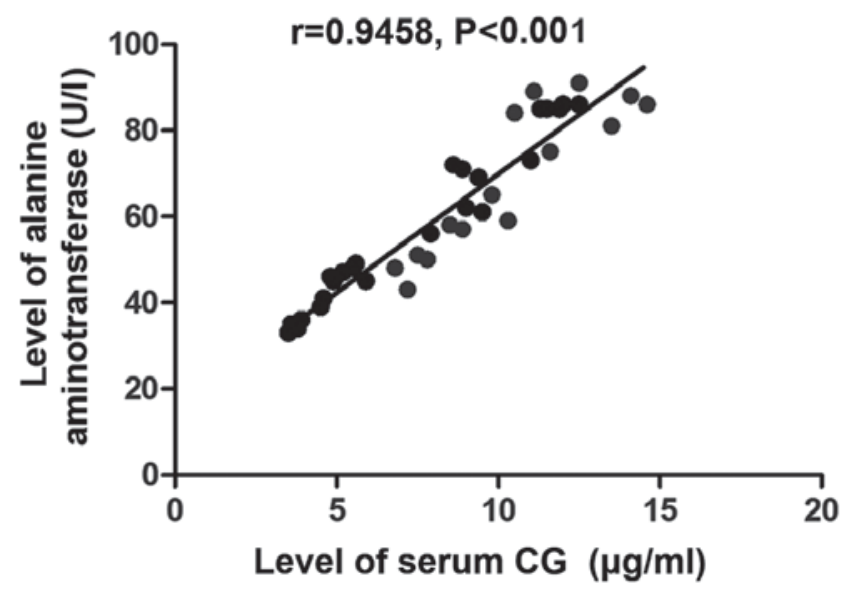

Figure 2. Spearman's correlation analysis of the level of serum CG in pregnant women with ICP with changes in the level of alanine aminotransferase. The level of serum CG in pregnant women with ICP is positively related to the level of alanine aminotransferase $(\mathrm{P}<0.05)$.

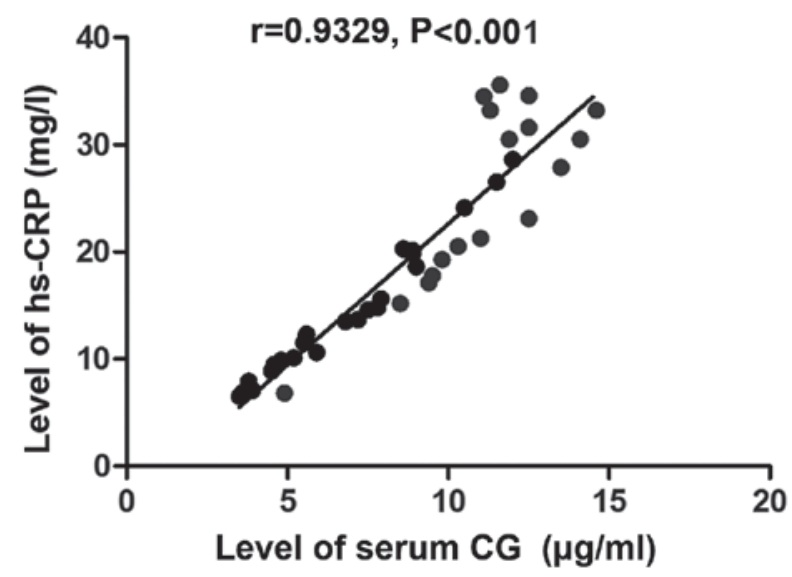

Figure 3. Spearman's correlation analysis of the level of serum CG in pregnant women with ICP with changes in the level of hs-CRP. The level of serum CG in pregnant women with ICP is positively correlated with the level of hs-CRP $(\mathrm{P}<0.05)$.

5 min after birth in the normal hepatic function group were evidently higher than those in the abnormal hepatic function group $(\mathrm{P}<0.05)$ (Table IV).

Changes in Apgar scores of newborns with different levels of inflammatory cytokines. Apgar scores of newborns at birth and at 1 and 5 min after birth in the normal inflammatory cytokine group were higher than those in the abnormal inflammatory cytokine group $(\mathrm{P}<0.05)$ (Table $\mathrm{V})$.

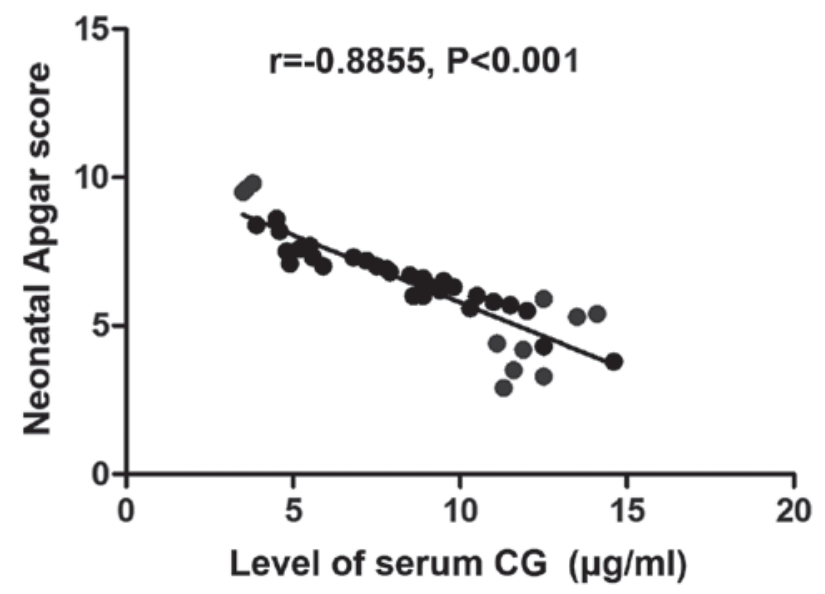

Figure 4. Spearman's correlation analysis of the level of serum CG in pregnant women with ICP with neonatal Apgar score. There is a negative correlation between the level of serum CG in pregnant women with ICP and neonatal Apgar score $(\mathrm{P}<0.05)$.

Correlation analyses of the serum CG level with umbilical artery $S / D$ ratio values in the third trimester of pregnancy during antenatal inspection, the level of alanine aminotransferase, the level of $h s-C R P$, neonatal Apgar score and gestational week. The level of serum CG in pregnant women with ICP was positively correlated with umbilical artery S/D ratio in the third trimester of pregnancy during antenatal inspection $(r=0.8508, P<0.001)$ (Fig. 1), the level of alanine aminotransferase $(\mathrm{r}=0.9458, \mathrm{P}<0.001)$ (Fig. 2) and the level of hs-CRP ( $\mathrm{r}=0.9329, \mathrm{P}<0.001)$ (Fig. 3), but negatively related to neonatal Apgar score $(\mathrm{r}=-0.8855, \mathrm{P}<0.001)$ (Fig. 4) and gestational week ( $\mathrm{r}=-0.8924, \mathrm{P}<0.001)$ (Fig. 5).

\section{Discussion}

ICP is a distinctive disease in the second and third trimesters of pregnancy, mainly manifested as pruritus, jaundice and abnormal hepatic function (10). A previous study (11) indicated that the high-level estrogen for a long term during pregnancy leads to excessive bile formation and acatharsia (12). Besides, selenium deficiency during pregnancy, ATP8B1 gene defects in pregnant women and positive cytochrome P450 1A2 (CYPIA2), an estrogen metabolism gene, are related factors contributing to ICP (13). ICP is harmful to the fetus and has a great adverse effect on the perinatal prognosis. The maternal cholestasis leads to abnormal liver function, which in turn leads to the decrease of normal endocrine function and toxin degradation ability of the liver (14). Over time, maternal bile acids and toxins are transferred to the fetus via placental circulation, leading to premature 
Table V. Changes in Apgar scores of newborns with different levels of inflammatory cytokines (point, mean \pm SD).

\begin{tabular}{lccc}
\hline Variables & At birth & At 1 min after birth & At 5 min after birth \\
\hline Normal inflammatory cytokine group & $8.3 \pm 0.4$ & $8.5 \pm 0.5$ & $9.2 \pm 0.3$ \\
Abnormal inflammatory cytokine group & $6.4 \pm 0.3$ & $6.7 \pm 0.4$ & $7.0 \pm 0.2$ \\
t value & 24.033 & 17.779 & 38.591 \\
P-value & $<0.001$ & $<0.001$ & $<0.001$ \\
\hline
\end{tabular}

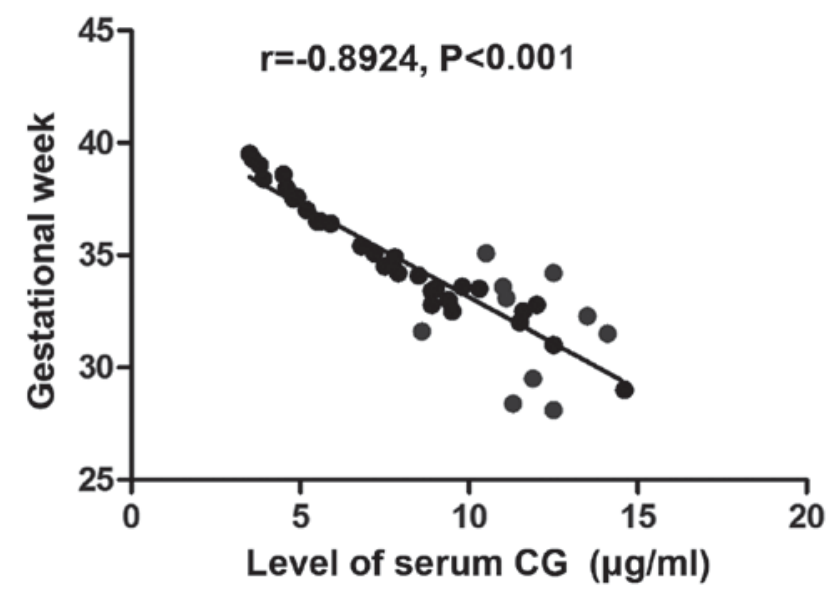

Figure 5. Correlation analysis of the level of serum CG in pregnant women with ICP with gestational week. There is a negative relationship between the level of serum CG in pregnant women with ICP and gestational week $(\mathrm{P}<0.05)$.

delivery, intrauterine distress and even fetal asphyxia (15). For the diagnosis and treatment of ICP during pregnancy, serum CG has the highest specificity, which can provide a better guidance for the clinical treatment. This study explored the effects of the level of serum CG in patients with ICP on hepatic function, inflammatory cytokines and fetal outcomes.

In the present study, all patients with cholestasis of pregnancy were treated with general prescription for ICP. Related conditions of newborns were compared between the two groups, and it was found, respectively, that birth weight, gestational week at birth and Apgar score at birth in the observation group were lighter, earlier and lower than those in the normal group, indicating that newborns delivered by these patients with ICP have lighter weight, earlier gestational week and lower birth Apgar scores. Additionally, comparisons of intrauterine and postpartum conditions between the two groups revealed that the incidence rates of grade II or above amniotic fluid contamination, intrauterine fetal distress and neonatal jaundice in the observation group were significantly higher than those in the normal group, further suggesting that the incidence rates of amniotic fluid contamination, intrauterine fetal distress and neonatal jaundice are obviously increased in patients with ICP. Comparison of the levels of inflammation-related cytokines between the two groups manifested that the levels of hs-CRP, IL-6, and TNF- $\alpha$ among the inflammation-related cytokines in the observation group detected at the time of inclusion were markedly higher than those in the control group, indicating that the levels of inflammatory cytokines in patients with normal hepatic function are significantly lower than those in patients with abnormal hepatic function. In addition, the study on changes in Apgar scores of newborns delivered by patients with different hepatic functions and different inflammatory cytokine levels demonstrated that Apgar scores of newborns at birth and at $1 \mathrm{~min}$ and $5 \mathrm{~min}$ after birth in the normal hepatic function and normal inflammatory cytokine groups were higher than those in the abnormal hepatic function group. This suggests that the hepatic function and the in vivo inflammatory cytokine level of pregnant women are associated with neonatal Apgar score to some extent. Finally, the correlation analyses of the serum CG level in pregnant women with umbilical artery $\mathrm{S} / \mathrm{D}$ ratio in the third trimester of pregnancy during antenatal inspection, the level of alanine aminotransferase, the level of hs-CRP, neonatal Apgar score and gestational week illustrated that the serum CG level in pregnant women was positively correlated with umbilical artery S/D ratio in the third trimester of pregnancy during antenatal inspection, the level of alanine aminotransferase and the level of hs-CRP, but negatively related to neonatal Apgar score and gestational week.

The high-level cholic acid, especially high $\mathrm{CG}$, in pregnant women with ICP, may cause the shrinkage of the placental villus (16), thus, resulting in edema of the placental trophocytes. Despite the increased number, edema occurs in the basal villus, and the villous cavity remarkably declines (17), thus causing a significant reduction of maternal blood flow in the placental villus cavity, fetal hypoxia and intrauterine distress (18). ICP in the body evidently stimulates fetal intestinal peristalsis to be enhanced (19), resulting in meconium-stained amniotic fluid, neonatal asphyxia and lowered neonatal Apgar score, so the proportions of contaminated amniotic fluid and intrauterine fetal distress in patients with ICP are increased. It was also verified in this study that the serum CG level in pregnant women was positively correlated with the level of alanine aminotransferase in hepatic function of pregnant women with ICP, umbilical artery $\mathrm{S} / \mathrm{D}$ ratio in the third trimester of pregnancy and the level of hs-CRP, but negatively related to neonatal Apgar score and gestational week. With the development of pregnancy, the placental circulatory resistance decreased, the end-diastolic blood flow rate accelerated, the umbilical blood flow resistance decreased, and the umbilical blood flow increased. However, in pregnant women with ICP, the number of arterioles in the third grade villous trunk of placenta decreased significantly, resulting in the obstruction of material exchange between fetus and mother through the umbilical cord, and the increase of $\mathrm{S} / \mathrm{D}$ value. Then the placental dysfunction affects the development of the fetus. In addition, neonatal jaundice mainly caused hypermetabolism of fetal hemoglobin catabolism due to intrauterine hypercholidemia and liver cell injury. After birth, microsomal and glucuronic acid binding disorder occurred 
in hepatocytes. Thus, the ability of bilirubin to decompose is reduced, and jaundice occurs in newborns (20). There was a positive correlation between serum cholic acid level and hs-CRP level in pregnant women with ICP. The increase of inflammatory cytokines in the body may lead to the increase of mitochondria and neutrophils, the increase of oxygen free radicals, the damage of lipid peroxidation and the increase of the level of immunological cytokines, which further aggravates the damage of liver function. It may also promote the production of a large amount of estrogen in the placenta, leading to the imbalance of estrogen and progesterone levels in the mother and placenta, further exacerbating ICP and increasing the level of serum glycine cholic acid (21).

In conclusion, in patients with ICP, as the level of CG in the body is increased, the levels of alanine aminotransferase and hs-CRP in pregnant women and umbilical artery $\mathrm{S} / \mathrm{D}$ ratio are significantly increased. Moreover, this leads to lower Apgar score of newborns, neonatal asphyxia, shortened gestational week and premature birth.

\section{Acknowledgements}

Not applicable.

\section{Funding}

No funding was received.

\section{Availability of data and materials}

The datasets used and/or analyzed during the current study are available from the corresponding author on reasonable request.

\section{Authors' contributions}

LW and ZL collected the general data of patients and were responsible for patient treatment. XZ and YD recorded and analyzed the observational indexes. LG helped with Apgar score analysis. All authors have read and approved the final manuscript.

\section{Ethics approval and consent to participate}

The study was approved by the Ethics Committee of Daqing Longnan Hospital (Daqing, China) and informed consents were signed by the patients and/or guardians.

\section{Patient consent for publication}

Not applicable.

\section{Competing interests}

The authors declare that they have no competing interests.

\section{References}

1. Estiú MC, Frailuna MA, Otero C, Dericco M, Williamson C, Marin JJG and Macias RIR: Relationship between early onset severe intrahepatic cholestasis of pregnancy and higher risk of meconium-stained fluid. PLoS One 12: e0176504, 2017.
2. Günaydin B, Bayram M, Altuğ M, Cevher S and Bozkurt N: Retrospective analysis of maternal, fetal, and neonatal outcomesof intrahepatic cholestasis of pregnancy at Gazi University. Turk J Med Sci 47: 583-586, 2017.

3. Kong X, Kong Y, Zhang F, Wang T and Zhu X: Expression and significance of dendritic cells and Th17/Treg in serum and placental tissues of patients with intrahepatic cholestasis of pregnancy. J Matern Fetal Neonatal Med 31: 901-906, 2018.

4. Tayyar AT, Kozalı S, Yetkin Yildirim G, Karakus R, Yuksel IT, Erel O, Neselioglu S and Eroglu M: Role of ischemia-modified albumin in the evaluation of oxidative stress in intrahepatic cholestasis of pregnancy. J Matern Fetal Neonatal Med 11: 1-5, 2018.

5. Sharifzadehgan S, Hermann M, Nedellec S, De Luca D and Benachi A: Intrahepatic cholestasis of pregnancy: Shorter duration of labor? Eur J Obstet Gynecol Reprod Biol 225: 258-259, 2018.

6. Feng C, Li WJ, He RH, Sun XW, Wang G and Wang LQ: Impacts of different methods of conception on the perinatal outcome of intrahepatic cholestasis of pregnancy in twin pregnancies. Sci Rep 8: 3985, 2018.

7. Wood AM, Livingston EG, Hughes BL and Kuller JA: Intrahepatic cholestasis of pregnancy: A review of diagnosis and management. Obstet Gynecol Surv 73: 103-109, 2018.

8. Covach AJ and Rose WN: Intrahepatic cholestasis of pregnancy refractory to multiple medical therapies and plasmapheresis. AJP Rep 7: e223-e225, 2017.

9. Floreani A and Gervasi MT: New insights on intrahepatic cholestasis of pregnancy. Clin Liver Dis 20 :177-189, 2016.

10. Koroglu N, Tayyar A, Tola EN, Yetkin Yildirim G, Temel Yuksel I, Aslan Cetin B, Dag I and Acar DK: Increased levels of the novel hepatokine fetuin $B$ in patients with intrahepatic cholestasis of pregnancy. J Matern Fetal Neonatal Med 12: 1-6, 2017.

11. Lin J, Gu W and Hou Y: Diagnosis and prognosis of early-onset intrahepatic cholestasis of pregnancy: a prospective study. J Matern Fetal Neonatal Med 7: 1-7, 2017.

12. Mei Y, Gao L, Lin Y, Luo D, Zhou X and He L: Predictors of adverse perinatal outcomes in intrahepatic cholestasis of pregnancy with dichorionic diamniotic twin pregnancies. J Matern Fetal Neonatal Med 32: 472-476, 2019.

13. Adeyemi O, Alvarez-Laviada A, Schultz F, Ibrahim E, Trauner M, Williamson C, Glukhov AV and Gorelik J: Ursodeoxycholic acid prevents ventricular conduction slowing and arrhythmia by restoring T-type calcium current in fetuses during cholestasis. PLoS One 12: e0183167, 2017.

14. Li L, Chen YH, Yang YY and Cong L: Effect of intrahepatic cholestasis of pregnancy on neonatal birth weight: A metaanalysis. J Clin Res Pediatr Endocrinol 10: 38-43, 2018.

15. Dadhwal V, Sharma A, Khoiwal K, Deka D, Sarkar P and Vanamail P: Pregnancy outcomes in HIV-infected women: Experience from a tertiary care center in India. Int $\mathrm{J}$ MCH AIDS 6: 75-81, 2017.

16. Maldonado M, Alhousseini A, Awadalla M, Idler J, Welch R, Puder K, Patwardhan M and Gonik B: Intrahepatic cholestasis of pregnancy leading to severe vitamin K deficiency and coagulopathy. Case Rep Obstet Gynecol 2017: 5646247, 2017.

17. Herrera CA, Manuck TA, Stoddard GJ, Varner MW, Esplin S, Clark EAS, Silver RM and Eller AG: Perinatal outcomes associated with intrahepatic cholestasis of pregnancy. J Matern Fetal Neonatal Med 31: 1913-1920, 2018.

18. Mattila M, Kemppainen H, Isoniemi $\mathrm{H}$ and Polo-Kantola P: Pregnancy outcomes after liver transplantation in Finland. Acta Obstet Gynecol Scand 96: 1106-1111,2017.

19. Tayyar A, Temel Yuksel I, Koroglu N, Tanay Tayyar A, Alici Davutoglu E, Akkaya Firat A and Aslan Cetin B: Maternal copeptin levels in intrahepatic cholestasis of pregnancy. J Matern Fetal Neonatal Med 31: 2066-2070, 2018.

20. Sanhal CY, Daglar K, Kara O, Y1lmaz ZV, Turkmen GG, Erel O, Uygur D and Yucel A: An alternative method for measuring oxidative stress in intrahepatic cholestasis of pregnancy: Thiol/ disulphide homeostasis. J Matern Fetal Neonatal Med 31: 1477-1482, 2018.

21. Türkmen GG, Timur H, Yilmaz Z, Kirbas A, Daglar K, Tokmak A, Uygur D and Danişman N: Effect of intrahepatic cholestasis of pregnancy on maternal serum screening tests. J Neonatal Perinatal Med 9: 411-415, 2016.

This work is licensed under a Creative Commons Attribution-NonCommercial-NoDerivatives 4.0 International (CC BY-NC-ND 4.0) License. 